\title{
Molecular assessment of allelic loss in Warthin tumors
}

\author{
Muammar Arida, E Leon Barnes and Jennifer L Hunt \\ Department of Pathology, University of Pittsburgh Medical Center, Pittsburgh, PA, USA
}

\begin{abstract}
Warthin's tumors are benign lesions of the head and neck that have a characteristic morphologic appearance. The etiology of Warthin's tumors is controversial and whether they are true neoplasms or developmental malformations continues to be debated. In this study, we examined 12 Warthin tumors with a molecular and immunohistochemical approach. Immunostains for p53 and p16ink were performed. The epithelial and lymphoid components of each lesion were microdissected and PCR was performed for $\mathbf{1 3}$ microsatellite markers at or near common tumor suppressor genes. The results were analyzed semiquantitatively using capillary electrophoresis. Frequency of allelic loss was calculated. The epithelial component of all tumors was negative for p53 and p16ink. By molecular genotyping there was only one case that had one locus with allelic imbalance, while the remainder had no evidence of clonal allelic loss. The immunohistochemical and molecular results in this study lend support to the hypothesis that Warthin tumors are non-neoplastic, as there was no evidence of aberrant staining for tumor suppressor gene protein products and no evidence of consistent clonal allelic losses.

Modern Pathology (2005) 18, 964-968. doi:10.1038/modpathol.3800379; Published online 29 April 2005
\end{abstract}

Keywords: Warthin tumor; loss of heterozygosity; clonality; neoplasm

Warthin's tumors are benign lesions of the head and neck that occur almost exclusively in the parotid gland or high cervical lymph nodes. They account for $4-11.2 \%$ of all salivary gland tumors. ${ }^{1,2}$

Warthin tumors are composed of an oncocytic epithelial component that can have a papillary, glandular-cystic, and/or solid growth pattern (Figure 1a). The lesions also have a surrounding dense lymphocytic infiltrate that can contain germinal centers. In studies of the lymphocytic population, a polyclonal pattern has been observed. ${ }^{3}$ The alternative name for these lesions reflects this histology: papillary cystadenoma lymphomatosum. These lesions can be multifocal and bilateral, and are more common in individuals who use tobacco. ${ }^{4,5}$

Although referred to as Warthin tumor, the etiology is controversial. Whether they are true neoplasms that arise as clonal proliferations, or whether they represent developmental malformations with a non-neoplastic origin continues to be an

Correspondence: Dr JL Hunt, MD, PUH A610.2, Department of Pathology, University of Pittsburgh Medical Center, 200 Lothrop Street, Pittsburgh, PA 15213, USA.

E-mail: HUNTJL@upmc.edu

This research was presented as a poster presentation at the United States and Canadian Academy of Pathology, February, 2004; Vancouver, BC, USA.

Received 29 July 2004; revised 6 December 2004; accepted 23 December 2004; published online 29 April 2005 issue. $^{6,7}$ Although several molecular studies have begun to address this controversy, the results have been conflicting. Classical cytogenetics studies have identified clonal abnormalities in some Warthin tumors. ${ }^{8-10}$ However, in another study, the epithelial component of the tumor was examined for clonality using the human androgen receptor assay (HUMARA). ${ }^{11}$ All informative cases showed a polyclonal X-inactivation pattern, which suggests that Warthin tumor is non-neoplastic.

In this study, we analyzed the epithelial component of Warthin tumor at the molecular level using assays that can detect allelic imbalance at or near known tumor suppressor genes. This clonal allelic imbalance is often found in neoplastic processes, with low-level allelic imbalance being typical of benign or low-grade tumors and high-level allelic imbalance being more typical of aggressive or malignant tumors. We also utilized immunohistochemical stains for two of the tumor suppressor genes studied, as a manner of validation of the PCR results, and also to look for isolated aberrant protein expression of p16ink4 and p53.

\section{Materials and methods}

The Institutional Review Board of the University of Pittsburgh approved this study (IRB \#0307086). Inclusion criteria for this study included the con- 
firmation of the diagnosis of Warthin tumor, and the availability of paraffin blocks. Cases were selected randomly from the archives of the Department of Pathology, Division of Anatomic Pathology of the University of Pittsburgh Medical Center. A paraffin tissue block of the lesion was selected to prepare additional slides for molecular testing.

Additional sections were obtained from the block as follows: one H\&E, eight unstained slides, and one final H\&E. The two H\&Es were examined to ensure that the tissue was not lost through sectioning. The last two unstained slides were reserved for immunohistochemical staining. Microdissection targets were selected on the first H\&E slide to include
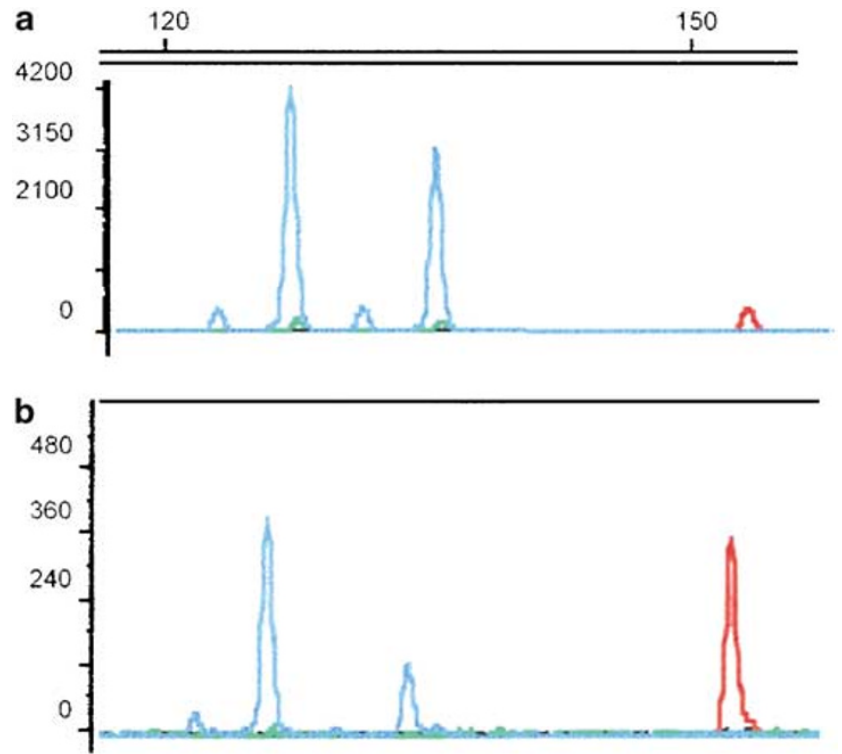

Figure 1 (a and b) Electropherograms for capillary electrophoresis using primers for D17s1289 in the one case that showed allelic imbalance (Case number 3). The lymphoid component shows balanced alleles, as the peak heights are within the normal range with a ratio of 1.33 (a). The peaks from the epithelial component show allelic imbalance, with a ratio of peak heights of $3.0(\mathbf{b})$. areas of pure epithelium and areas of adjacent normal salivary tissue. The microdissection targets ranged from 3 to $5 \mathrm{~mm}$ in diameter. The six unstained slides were microdissected, using the marked $\mathrm{H} \& \mathrm{E}$ as a guide, under direct visualization using a stereoscopic microscope. The microdissected tissue fragments were placed into lysis buffer and digested using proteinase K. A DNA extraction was subsequently performed using the QIAamp DNA mini kit extraction column (Qiagen, Valencia, CA, USA).

Polymerase chain reaction (PCR) primers were used to amplify short tandem repeat units that colocalize with known tumor suppressor genes that have been reported to be affected in salivary gland neoplasms (Table 1). A semiquantitative analysis was performed using capillary electrophoresis (Applied Biosystems, Inc., Prism 3100 and Genescan software). The normal tissue was examined first, to determine whether the markers were informative (ie, patient is heterozygous for the short tandem repeat unit). Then, the DNA obtained from the epithelium of the lesion was examined for these informative markers to detect allelic imbalance, which correlates with loss of heterozygosity. For this analysis, the height of the peaks representing the PCR product relative fluorescence is used. When the ratio of the peak heights for normal compared to tumor is $<0.5$ or $>2.0$, this is indicative of allelic imbalance. A frequency of allelic loss (FAL) is obtained by dividing the overall number of markers with allelic loss by the number of markers that were informative.

Immunohistochemical stains were performed for p16INK4 (BD PharMingen, San Diego, CA, USA, G175-405 clone, 1:200 dilution) and p53 (Dako, Carpenteria, CA, USA, DO-7 clone, 1:200 dilution). Antigen retrieval was performed using a pressure cooker pretreatment and subsequent cooling. The antibody staining was performed using a standard streptavadin-biotin approach. Positive (lung carcinoma for p53 and cervical carcinoma for p16INK4) and negative controls were adequate. The slides

Table 1 This table details the tumor suppressor genes, chromosomal locations, markers utilized, the primer sequences for all loci examined in this study

\begin{tabular}{|c|c|c|c|c|c|}
\hline Tumor suppressor gene & Chromosome location & Marker & Forward primer $\left(5^{\prime} \rightarrow 3^{\prime}\right)$ & Reverse primer $\left(5^{\prime} \rightarrow 3^{\prime}\right)$ & $F A L$ \\
\hline$L-M Y C$ & $1 \mathrm{p} 34.2$ & MYCL.5NT & ccgtagcctggcgagactcc & gaaaattcgacgttgttaaag & $0 / 9$ \\
\hline$C M M$ & $1 \mathrm{p} 36.2$ & D1s.407 & gcctgtgctaaccacatgg & gacccgccccctatcttcc & $0 / 10$ \\
\hline hOGG1 & $3 p 26$ & D3s.2303 & gttagtatcccaaggggagc & ggactgggacagaggtctcg & $0 / 10$ \\
\hline VHL & 3 p26 & D3s.1539 & ccattactctctccatagctag & gaaaatacattacttcgac & $0 / 5$ \\
\hline$A P C$ & $5 q 21$ & D5s.615 & gagtcagggttttacagag & ggaaaagtaaacagataca & $0 / 8$ \\
\hline MCC & $5 q 21$ & D5s.592 & ggcctaactggaatgtgt & cgggtctatttgtggtcg & $0 / 1$ \\
\hline РТСH1 & $9 q 22.1$ & D9s.252 & ttgtcaactcctaatatggac & catactcttgaacccctatag & $0 / 8$ \\
\hline$M T S 1 / p 16$ (a) & $9 \mathrm{p} 21$ & D9s.251 & tattctgcatgttttatgtg & gaaaaattccgaaacatc & $0 / 5$ \\
\hline MTS1/p16 (b) & 9p21 & D9s.254 & tatcctgggtaataactgcc & ctcctatttggacgaagtgag & $0 / 9$ \\
\hline p53 (a) & 17p13 & D17s.1289 & agcctgggcaataagagctg & gggtgaaaagacaacaaac & $1 / 8$ \\
\hline p53 (b) & $17 \mathrm{p} 13$ & D17s.974 & cgcggttgtttcattccg & gacacacgtgaccgcgacac & $0 / 10$ \\
\hline NF2 (a) & $22 \mathrm{q} 12.2$ & D22s.417 & tatgacacggcaagaccc & tccaаtcaасcaccaac & $0 / 7$ \\
\hline NF2 (b) & $22 q 12.2$ & D22s.532 & gcctgggcaacagagcgag & cttcccaaggaaaaccttaac & $0 / 7$ \\
\hline
\end{tabular}

When several primer sets were utilized for the same gene, they are listed as $a$ and $b$. The FAL is also shown for each maker for all tumors studied. 
were examined to identify positive nuclear and/or cytoplasmic staining. The intensity of staining was graded on a scale of $0-4(0=$ negative, $4=$ strongly positive) and the percentage of the epithelial staining was estimated.

\section{Results}

The clinical data from the 12 cases are presented in Table 2. The tumors arose in the parotid gland in all cases. There were four males and eight females with an average age of 60 years (range 47-76). The average tumor size was $2.45 \mathrm{~cm}$ (range 1.1-4.2). There was a known history of tobacco use in $\frac{5}{12}$ cases. Two of the patients had multiple lesions, but none were bilateral and none of the tumors recurred.

DNA extraction and PCR were successful in all cases. The average number of informative loci for each case was 9. Overall, there was only one locus identified with allelic imbalance, and this occurred in the epithelial component of only one lesion (which can be seen in Table 2 and Figure 1). The lymphoid component, which was used as a normal control, did not exhibit allelic imbalance in any of the cases. No allelic imbalance was seen in all other lesions.

Immunohistochemical stains for p53 and p16ink were negative in all cases (Figure $2 b$ and $c$ ). This was compared to the strong nuclear stain present in the positive control for p53 stain and the strong cytoplasmic staining seen in the positive control for the p16ink.

\section{Discussion}

Warthin tumors are common entities that have a characteristic histologic appearance. The epithelial component has a distinctive oncocytic papillary-cystic appearance associated with a lymphoid background, often with germinal centers. The tumors can be multiple and/or bilateral, and can even occur outside of the parotid gland in high cervical lymph nodes or salivary gland rests, but this is never considered to be evidence of metastatic disease. $^{2}$ In very rare instances, malignancy can arise in conjunction with a Warthin's tumor, either in the form of lymphoma or carcinoma. ${ }^{12-14}$ It remains controversial whether these are merely synchronous lesions, or are related pathogenetically. The extremely low rate of malignancy, as compared to that seen in pleomorphic adenoma (carcinoma ex pleomorphic adenoma) suggests that the tumors are synchronous rather than clonally related.

The etiology of Warthin tumor has been long disputed. Whether it is a true neoplasm or a nonneoplastic developmental abnormality is unclear, despite several attempts even at the molecular level to settle this question. In fact, only rare studies have shown clonal genetic abnormalities, while most have not. $^{7-9,11}$ In one study, a translocation, $\mathrm{t}(11 ; 19)(\mathrm{q} 21 ; \mathrm{p} 13.1)$ was identified in a single Warthin's tumor. ${ }^{10}$ Since the cultured tumor contained both the epithelial and lymphoid cellular components, the authors report that they could not rule out the possibility that the translocation was contributed by the lymphoid component of the tumor. Another cytogenetics study detected several other translocations and some structural abnormalities in seven of 12 Warthin's tumors, though this included loss of the $\mathrm{Y}$ chromosome, which some regard as a normal phenomenon in aging. ${ }^{8}$ One of their tumors had a translocation that also involved chromosome 11. A study that argued against abnormalities in the epithelial component demonstrated that false aneuploidy was found by DNA flow cytometry that was attributed to contaminating lymphocytes. ${ }^{7}$ In another study arguing against clonality in Warthin's tumors, the authors performed X-inactivation studies using the HUMARA assay on the epithelial component of Warthin's tumors from women. Their results demonstrated a polyclonal X-inactivation pattern in all seven cases that were heterozygous for the HUMARA locus. ${ }^{11}$

The lymphoid infiltrate in Warthin's tumors has also been definitively shown to be polyclonal and non-neoplastic in prior work, which was supported

Table 2 This table gives the clinical and pathologic characteristics and the FAL for each case that was included in this study

\begin{tabular}{|c|c|c|c|c|c|c|}
\hline Case & Age (years) & Gender & Location & Size $(\mathrm{cm})$ & Tobacco & $F A L(\%)$ \\
\hline 1 & 73 & $\mathrm{~F}$ & Right parotid & 2 & N/A & 0 \\
\hline 2 & 61 & $\mathrm{~F}$ & Right parotid & 2 & Yes & 0 \\
\hline 3 & 50 & $\mathrm{~F}$ & Right parotid & 2.1 & Yes & 14 \\
\hline 4 & 58 & $\mathrm{~F}$ & Left parotid & 4 & N/A & 0 \\
\hline 5 & 57 & $\mathrm{~F}$ & Right parotid & 1.1 & N/A & 0 \\
\hline 6 & 53 & $\mathrm{~F}$ & Left parotid & 2 & Yes & 0 \\
\hline 7 & 58 & M & Left parotid & Three lesions $1.0-2.0$ & N/A & 0 \\
\hline 8 & 76 & $\mathrm{~F}$ & Right Parotid & 3 & Yes & 0 \\
\hline 9 & 70 & $\mathrm{~F}$ & Left parotid & 2 & Yes & 0 \\
\hline 10 & 56 & M & Left parotid & 4.2 & N/A & 0 \\
\hline 11 & 58 & $\mathrm{M}$ & Left parotid & Two lesions 1.2, 0.6 & N/A & 0 \\
\hline 12 & 47 & M & Left parotid & 3.8 & N/A & 0 \\
\hline
\end{tabular}

N/A indicates that the data were not available. 

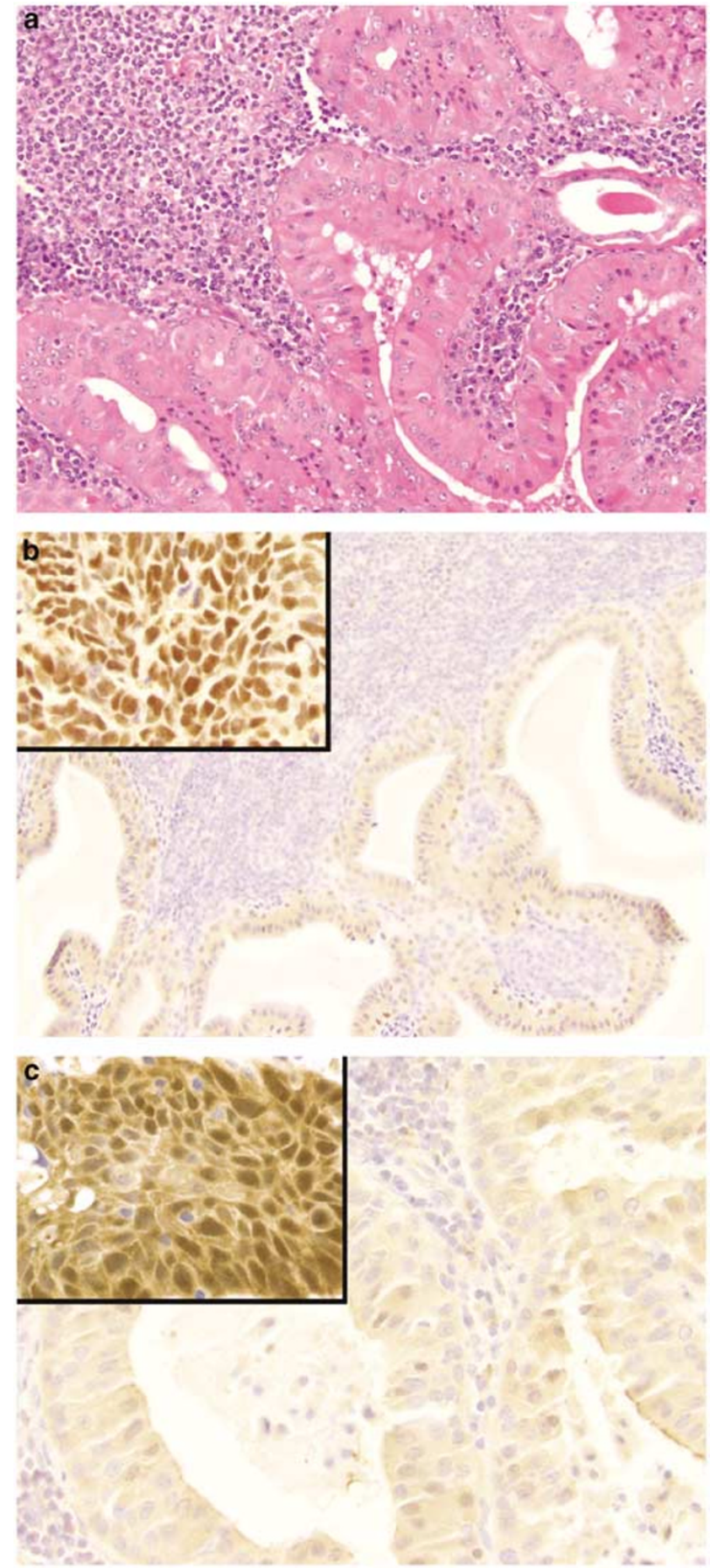

Figure 2 (a) $(\times 200$ original magnifications $)$ This is a photomicrograph of the H\&E stained slide from a Warthin tumor. The oncocytic epithelium and the surrounding lymphoid stroma can be appreciated. (b) ( $\times 100$ original magnification) This is a photomicrograph of the negative p53 stain. The inset demonstrates the positive control. (c) ( $\times 400$ original magnification) This is a photomicrograph of the negative p16ink stain. The inset demonstrates the positive control.

by our findings that the lymphoid component was completely normal by genotyping analysis. ${ }^{3}$ One possible explanation of the rare finding of cytoge- netic abnormalities is that these lesions do occasionally undergo neoplastic transformation secondary to proliferative stress.

In this study, we sought to determine whether there was clonality of Warthin tumors by examining a panel of tumor suppressor genes for loss of heterozygosity. In low-grade and benign neoplastic processes, it is typical for low-level allelic imbalance to be present, while in higher-grade malignant processes, there may be high-level allelic imbalance. Interestingly, oncocytic neoplasms of other organs often have a particularly high mutational rate. ${ }^{15}$ If the oncocytic epithelium of Warthin tumor were neoplastic, we expected to find a discernible level of clonal allelic losses.

Another method to assess tumor suppressor genes is to examine the protein expression patterns for the gene products. Aberrant expression of these protein products has been shown to correlate with both genetic and epigenetic changes in benign and malignant neoplastic processes. In this study, we examined Warthin tumors with immunohistochemical stains for two of the genes that were studied: p16ink and p53. This was performed as confirmation of the genetic results, and also to detect any aberrant epigenetic changes in the protein products from these two tumor suppressor genes.

Our results demonstrated negligible to absent clonal allelic losses across a broad spectrum of markers for tumor suppressor genes. In fact, only one allelic loss was found in the epithelium of the lesions, and no allelic imbalance was found in the lymphoid component. The immunohistochemistry results for protein expression paralleled those found at the DNA level. There was no overexpression of either p53 or of p16ink in these cases.

Overall, our results indicate that there are negligible clonal alterations at the genomic level or at the protein expression level for a variety of common tumor suppressor genes. Furthermore, because the detection of allelic loss in microdissected samples is also an indication of clonal expansion, we also believe that these data argue against a clonal process and lend support to the hypothesis that Warthin tumors are non-neoplastic. Additional work to compare tumors that are multifocal or bilateral would be important, as those lesions could potentially have a different pathogenesis.

\section{References}

1 Maiorano E, Lo Muzio L, Favia G, et al. Warthin's tumour: a study of 78 cases with emphasis on bilaterality, multifocality and association with other malignancies. Oral Oncol 2002;38:35-40.

2 Yoo GH, Eisele DW, Askin FB, et al. Warthin's tumor: a 40-year experience at The Johns Hopkins Hospital. Laryngoscope 1994;104:799-803.

3 Takezawa K, Jackson C, Gnepp DR, et al. Molecular characterization of Warthin tumor. Oral Surg Oral Med Oral Pathol 1998;85:569-575. 
4 Pinkston JA, Cole P. Cigarette smoking and Warthin's tumor. Am J Epidemiol 1996;144:183-187.

5 Kotwall CA. Smoking as an etiologic factor in the development of Warthin's tumor of the parotid gland. Am J Surg 1992;164:646-647.

6 Ohmori T, Uraga N, Tabei R. Warthin's tumor as a hamartomatous dysplastic lesion: a histochemical and immunohistochemical study. Histol Histopathol 1991; 6:559-565.

7 Banez EI, Krishnan B, Ansari MQ, et al. False aneuploidy in benign tumors with a high lymphocyte content: a study of Warthin's tumor and benign thymoma. Hum Pathol 1992;23:1244-1251.

8 Martins C, Fonseca I, Roque L, et al. Cytogenetic characterisation of Warthin's tumour. Oral Oncol 1997; 33:344-347.

9 Mark J, Dahlenfors R, Stenman G, et al. Chromosomal patterns in Warthin's tumor. A second type of human benign salivary gland neoplasm. Cancer Genet Cytogenet 1990;46:35-39.
10 Bullerdiek J, Haubrich J, Meyer K, et al. Translocation $\mathrm{t}(11 ; 19)(\mathrm{q} 21 ; \mathrm{p} 13.1)$ as the sole chromosome abnormality in a cystadenolymphoma (Warthin's tumor) of the parotid gland. Cancer Genet Cytogenet 1988;35:129-132.

11 Honda K, Kashima K, Daa T, et al. Clonal analysis of the epithelial component of Warthin's tumor. Hum Pathol 2000;31:1377-1380.

12 Podlesak T, Doleckova V, Sibl O. Malignancy of a cystadenolymphoma of the parotid gland. Eur Arch Otorhinolaryngol 1992;249:233-235.

13 Bunker ML, Locker J. Warthin's tumor with malignant lymphoma. DNA analysis of paraffin-embedded tissue. Am J Clin Pathol 1989;91:341-344.

14 Banik S, Howell JS, Wright DH. Non-Hodgkin's lymphoma arising in adenolymphoma-a report of two cases. J Pathol 1985;146:167-177.

15 Erickson LA, Jalal SM, Goellner JR, et al. Analysis of Hurthle cell neoplasms of the thyroid by interphase fluorescence in situ hybridization. Am J Surg Pathol 2001;25:911-917. 